\title{
Biochemical aspects of bacterial strategies for handling the incomplete translation processes
}

\author{
Yoshihiro Shimizu* \\ Laboratory for Cell-Free Protein Synthesis, Quantitative Biology Center - RIKEN, Kobe, Hyogo, Japan
}

\section{Edited by:}

Kenneth C. Keiler, Pennsylvania State University, USA

\section{Reviewed by:}

Jason Warren Cooley, University of

Missouri, USA

Tanel X. Tenson, University of Tartu, Estonia

\section{*Correspondence:}

Yoshihiro Shimizu, Laboratory for

Cell-Free Protein Synthesis,

Quantitative Biology Center - RIKEN

2-2-3 Minatojima-minamimachi,

Chuo-ku, Kobe, Hyogo 650-0047,

Japan

e-mail: yshimizu@riken.jp
During protein synthesis in cells, translating ribosomes may encounter abnormal situations that lead to retention of immature peptidyl-tRNA on the ribosome due to failure of suitable termination processes. Bacterial cells handle such situations by employing three systems that rescue the stalled translation machinery. The transfer messenger RNA/small protein B (tmRNA/SmpB) system, also called the trans-translation system, rescues stalled ribosomes by initiating template switching from the incomplete mRNA to the short open reading frame of tmRNA, leading to the production of a protein containing a C-terminal tag that renders it susceptible to proteolysis. The ArfA/RF2 and ArfB systems rescue stalled ribosomes directly by hydrolyzing the immature peptidyl-tRNA remaining on the ribosome. Here, the biochemical aspects of these systems, as clarified by recent studies, are reviewed.

Keywords: tmRNA, SmpB, ArfA, RF2, ArfB, trans-translation, ribosome rescue, quality control

\section{INTRODUCTION}

During cellular protein synthesis, ribosomes interact with a variety of macromolecules to enable the precise translation of genetic information into functional polypeptides. One of the most frequent ribosomal interactions is the process of sense codon decoding, in which a codon of $\mathrm{mRNA}$ interacts with an anticodon of tRNA in the A site of the ribosome. In bacteria, elongation factor $\mathrm{Tu}(\mathrm{EF}-\mathrm{Tu})$ plays a crucial role in this process by delivering aminoacyl-tRNA to the ribosomal A site as part of a ternary complex (aminoacyl-tRNA/EF-Tu/GTP). When the anticodon of aminoacyl-tRNA in the ternary complex matches the mRNA codon in the ribosomal A site, GTP hydrolysis is stimulated and EF-Tu changes its conformation, leading to dissociation of the EF-Tu/GDP binary complex, accommodation of aminoacyl-tRNA at the A site of the ribosome, and transfer of a nascent polypeptide to the aminoacyl-tRNA.

Although its molecular mechanism is similar to that of sense codon decoding, termination codon decoding does not require both tRNA and EF-Tu. In this process, a termination codon of mRNA interacts with class- 1 release factors (RFs) including RF1 and RF2, at the A site of the ribosome. Like the anticodon of tRNA in sense codon decoding, a specific region of class-1 RFs, known as a peptide anticodon (PAT motif for RF1 and SPF motif for RF2; Ito et al., 2000), recognizes and interacts directly with the mRNA termination codon in the decoding center of the ribosomal A site. Class-1 RFs can be accommodated at the ribosomal A site without the requirement for factors such as EF-Tu. Unlike aminoacyl-tRNA accommodated at the A site, class-1 RFs do not receive the nascent polypeptide of peptidyl-tRNA in the ribosomal P site but catalyze peptidyl-tRNA hydrolysis through a specific GGQ amino acid motif (Frolova etal., 1999; Song etal., 2000). Recent structural analyses of complexes composed of a ribosome and class-1 RFs have provided evidence for these molecular mechanisms (Korostelev et al., 2008; Laurberg et al., 2008; Korostelev, 2011).

Bacterial cells have evolved processes that enable ribosomes to progress in the absence of codon decoding. Because these processes generally take place when a ribosome is abnormally stalled by the lack of binding of a molecule to the A site, they are termed ribosome rescue processes. Ribosome stalling is most commonly caused by the lack of an in-frame termination codon in a mRNA, which can occur as a result of digestion of the mRNA (Chadani etal., 2011a; Garza-Sánchez et al., 2011), termination of transcription before the termination codon (Abo et al., 2000; Kobayashi et al., 2008), or misreading of the termination codon (Ueda etal., 2002). In these situations, the ribosome typically reaches the $3^{\prime}$-end of mRNA and translation is stopped by the absence of a codon in the A site. Abortive pausing of translation at certain regions of mRNAs can also occur as a result of ribosome stalling caused by the occurrence of a rare codon cluster (Roche and Sauer, 1999), amino acid starvation (Garza-Sánchez et al., 2008; Li et al., 2008), or the presence of specific sequences that cause elongation arrest (Sunohara etal., 2004). In these situations, immature peptidyl-tRNA remains on the ribosome and the resultant ribosome/mRNA/peptidyl-tRNA complex is stable enough to inhibit ribosome recycling; therefore, ribosome rescue processes are essential for its recovery.

In bacteria, the transfer messenger $\mathrm{RNA} /$ small protein $\mathrm{B}$ (tmRNA/SmpB) system (also called the trans-translation system), the alternative ribosome rescue factor A (ArfA)/RF2 system, and the alternative ribosome rescue factor $\mathrm{B}(\mathrm{ArfB})$ system are involved in the rescue of stalled ribosomes. This review focuses on the biochemical aspects of these three processes, as elucidated by recent studies. 


\section{THE TRANS-TRANSLATION SYSTEM}

The trans-translation system is a bypass process for the translation machinery. When a ribosome lacks an in-frame termination codon, translation is stalled at the $3^{\prime}$ end of the mRNA. Subsequently, tmRNA, a unique molecule that acts as both a tRNA and an mRNA, recognizes the stalled ribosome/mRNA/peptidyltRNA complex and is accommodated at the ribosomal A site as alanyl-tmRNA. After A site entry, translation is switched from the incomplete mRNA to the open reading frame (ORF) of the tmRNA, which contains an authentic translation termination codon. The tmRNA-encoded peptide sequence attached to the C-terminus of newly synthesized proteins, known as an SsrA tag, acts as a degradation signal for proteolysis. Through this process, stalled ribosomes are recycled for new translation reactions and the SsrA-tagged proteins are degraded immediately, which may contribute to the maintenance of both the translation process and the quality of cellular proteins (Figure 1).

\section{THE STRUCTURE AND FUNCTION OF tmRNA}

Escherichia coli tmRNA consists of 363 nucleotides and contains a tRNA-like domain (TLD), an ORF region encoding an SsrA tag, and four pseudoknot structures (PK1-4; Figure 2). One of the characteristic features of E. coli tmRNA is the TLD, in which the $5^{\prime}$

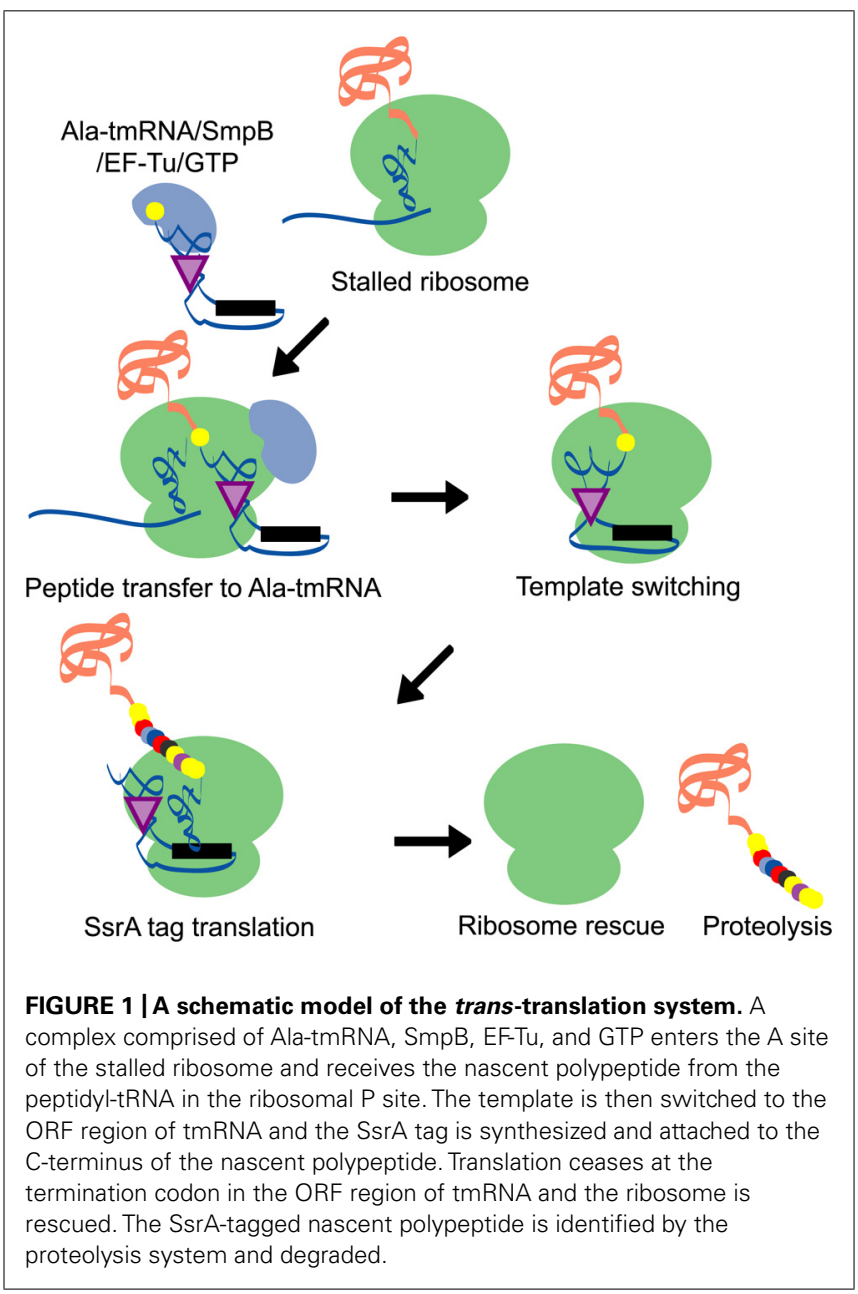

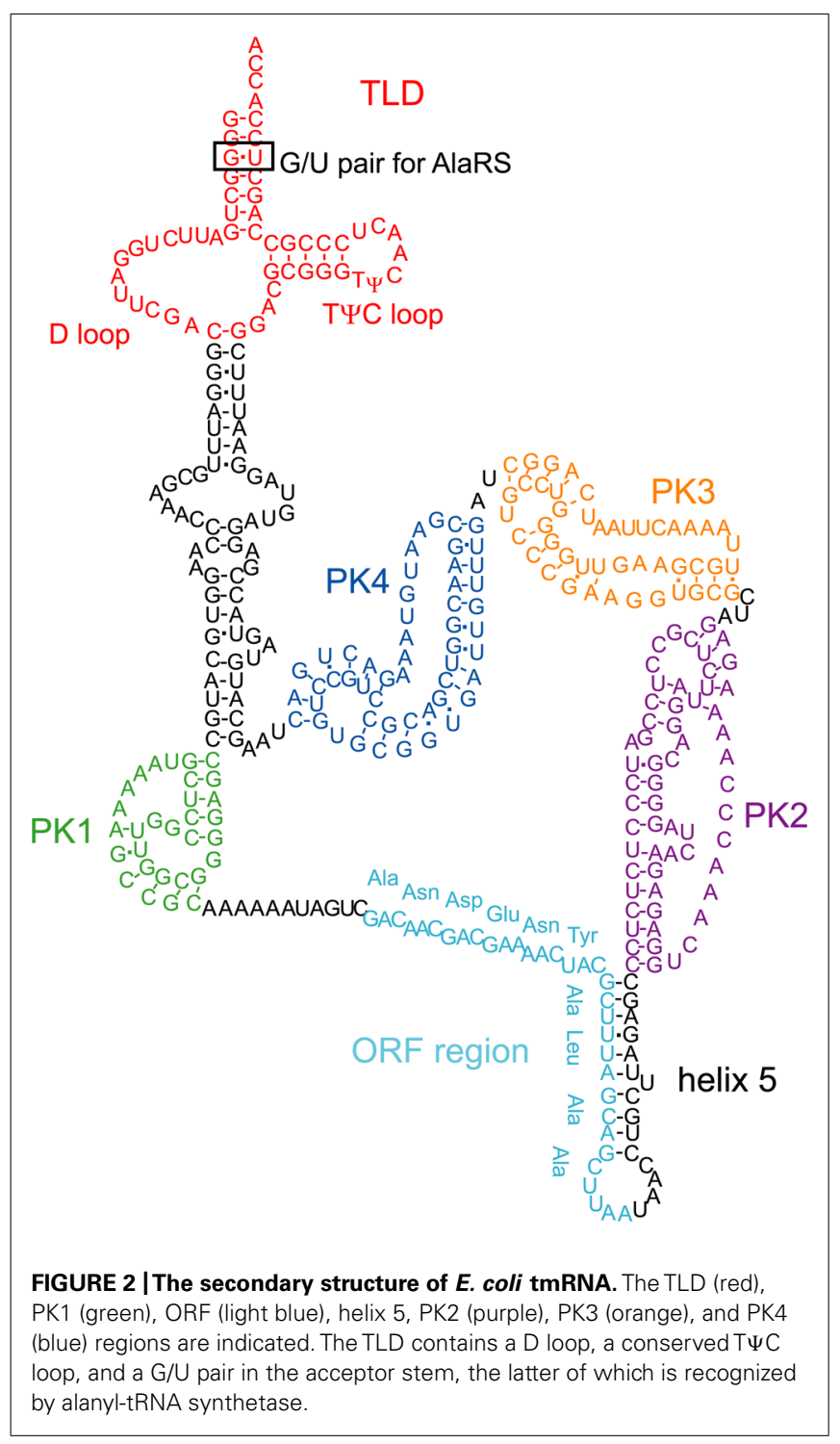

and $3^{\prime}$ terminal regions of tmRNA combine to form a tRNA-like structure. Like standard tRNA, tmRNA has an acceptor stem with a $3^{\prime}$-CCA end, as well as a T $\Psi \mathrm{C}$ stem and loop structure that includes conserved bases. These features of tmRNA not only facilitate its recognition and processing by RNase P (Komine et al., 1994), but also allow it to accept nucleotide modifications such as thymine and pseudouridine in the T $\Psi \mathrm{C}$ loop (Felden et al., 1998) in a manner similar to that of standard tRNA. Moreover, the acceptor stem region of tmRNA has a $\mathrm{G} / \mathrm{U}$ wobble base pair, which is a determinant base pair in the acceptor stem of tRNA $^{\text {Ala }}$ for recognition by alanyl-tRNA synthetase (Hou and Schimmel, 1988; Francklyn and Schimmel, 1989). Accordingly, tmRNA can undergo alanylation by alanyl-tRNA synthetase (Komine et al., 1994).

The ORF region of E. coli tmRNA is located downstream of the first pseudoknot structure (PK1) and encodes a peptide with the sequence ANDENYALAA (Figure 2). Together with the alanine residue attached by tmRNA itself, the AANDENYALAA peptide forms an SsrA degradation signal tag. The YALAA portion of 
the tag is important for degradation by ClpXP or ClpAP protease (Gottesman et al., 1998; Kim et al., 2000). In addition, the asparagine residue at the third position of the SsrA tag is important for recognition by $\mathrm{SspB}$ (stringent starvation protein $\mathrm{B}$ ), an adaptor protein that stimulates the degradation of tagged proteins by ClpXP (Levchenko et al., 2000; Lies and Maurizi, 2008).

Other structural elements of tmRNA also affect its function. The region containing PK1 located upstream of the ORF is important for the trans-translation reaction (Nameki et al., 1999), although formation of the pseudoknot structure is not absolutely necessary (Tanner et al., 2006; Wower et al., 2009). Furthermore, disruption of the three pseudoknot structures (PK2-4) located downstream of the ORF and the helix 5 region that contains the partial ORF sequence also impair tmRNA function, even though these regions are not indispensable (Nameki et al., 2000; Wower et al., 2004).

\section{THE ADAPTER PROTEIN SmpB IS ESSENTIAL FOR tmRNA FUNCTION}

The adapter protein SmpB is essential for the overall functions of tmRNA in the trans-translation process. SmpB was originally discovered as a protein whose gene is tandemly arranged with the ssr A gene (encoding tmRNA) in E. coli and Salmonella typhimurium. Deletion of the $s m p B$ gene in E. coli produces a phenotype identical to that of $s s r A$-defective cells and SmpB binds specifically to tmRNA with high affinity (Karzai et al., 1999).

NMR (nuclear magnetic resonance) analyses of SmpB from Aquifex aeolicus and Thermus thermophilus showed that the proteins consist of a globular core domain with a flexible C-terminal (C-tail) region (Dong et al., 2002; Someya et al., 2003). The core domain forms an oligonucleotide-binding fold (Murzin, 1993) that is similar to several RNA-binding proteins associated with the translation machinery, including ribosomal protein S17, initiation factor 1 (IF1), and the N-terminal domain of aspartyl-tRNA synthetase (Draper and Reynaldo, 1999).

The core domain and the C-tail region of SmpB play distinct roles in the trans-translation process. Consistent with its oligonucleotide-binding fold configuration, the core domain of SmpB plays a role in the interaction with tmRNA. In the crystal structure of the complex composed of the entire TLD of tmRNA and SmpB from T. thermophilus (Bessho et al., 2007), SmpB binds to the bottom of the D loop and T $\Psi \mathrm{C}$ stem of tmRNA; this region corresponds to the $\mathrm{D}$ stem and anticodon arm of the $\mathrm{L}$ shaped tRNA (Figure 3). Binding of SmpB to the TLD of tmRNA contributes to the formation of an L-shaped structure by compensating for the lack of a D stem in tmRNA and thereby assisting $\mathrm{D}$ and $\mathrm{T} \Psi \mathrm{C}$ arm interaction, and by stabilizing the coaxial structure of the T $\Psi \mathrm{C}$ and acceptor stems (Bessho et al., 2007). Thus, the core domain of SmpB and the TLD of tmRNA cooperatively mimic canonical tRNA in a sophisticated manner (Figure 3). SmpB enhances alanylation of tmRNA or the TLD by alanyl-tRNA synthetase (Barends et al., 2001; Hanawa-Suetsugu et al., 2002; Shimizu and Ueda, 2002, 2006) and promotes protection of the aminoacyl moiety of alanyl-TLD by EF-Tu (Shimizu and Ueda, 2006). Stabilization of the acceptor arm region by SmpB may contribute to these effects because both alanyl-tRNA synthetase and EF-Tu interact with the acceptor arm of tRNA (Nissen et al., 1995; Swairjo et al., 2004).

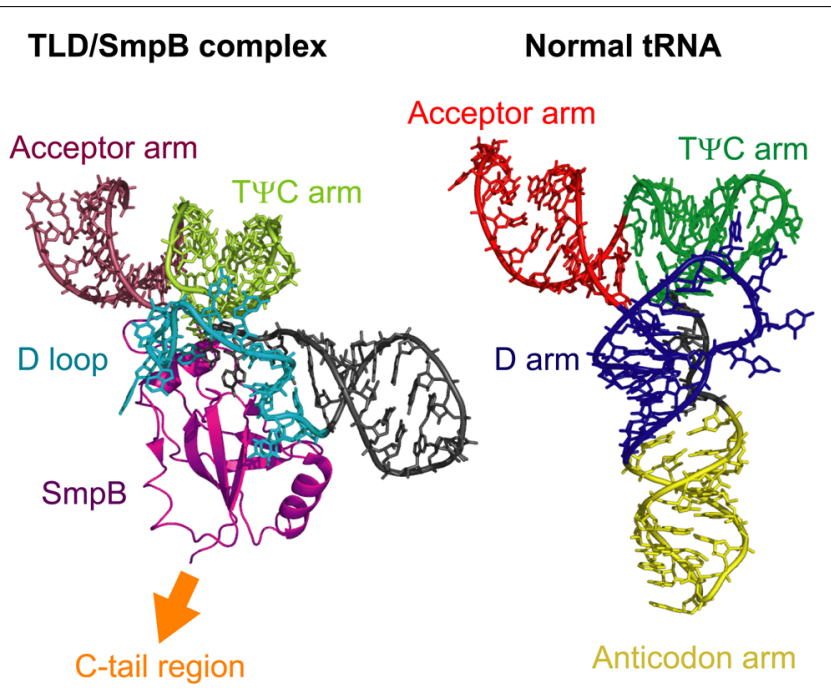

FIGURE 3 | A comparison of the structures of the TLD/SmpB complex and normal tRNA. The left panel shows the crystal structure of the complex comprised of the TLD of tmRNA and SmpB from Thermus thermophilus and the right panel shows that of normal tRNA ( $E$. coli tRNA ${ }^{C y s}$ ). The acceptor arm, T $\Psi C$ arm, D loop or D arm, and anticodon arm, or SmpB are indicated. The $\mathrm{C}$-tail region of SmpB extends to the opposite side of the TLD. The structural coordinates for the TLD/SmpB and normal tRNA complexes were taken from PDB entries 2CZJ (Bessho et al., 2007) and 1B23 (Nissen etal., 1999), respectively.

In the crystal structure of the tmRNA/SmpB complex, the C-tail region of SmpB extends to the opposite side of the TLD and is positioned in a proximal region corresponding to the anticodon loop of canonical L-shaped tRNAs (Figure 3). This arrangement locates the $\mathrm{C}$-tail region of $\mathrm{SmpB}$ close to the decoding region of the $30 \mathrm{~S}$ ribosomal subunit during entry of tmRNA into the ribosomal A site (Kaur et al., 2006). Furthermore, deletion of more than seven amino acids from the C-terminus of this region inhibits the addition of the SsrA tag to the nascent polypeptide in vivo (Jacob et al., 2005; Sundermeier et al., 2005) or alanyl-transfer to the nascent polypeptide on the ribosome in vitro (Shimizu and Ueda, 2006), without affecting the alanylation efficiency of tmRNA. In addition, several mutations in the conserved residues of the C-tail region have similar inhibitory effects (Sundermeier et al., 2005; Miller et al., 2011). These studies indicate that the C-tail region of SmpB plays a crucial role in the trans-translation process through an interaction with the decoding region of the $30 \mathrm{~S}$ ribosomal subunit.

\section{THE TRANS-TRANSLATION PROCESS ON THE RIBOSOME}

During the trans-translation process, the alanyl-tmRNA/SmpB complex can enter the A site of the ribosome without the requirement for codon-anticodon interaction. The mechanisms involved in sense codon decoding in the canonical translation system have been well-characterized by kinetic experiments (Rodnina and Wintermeyer, 2001; Daviter et al., 2006), cryoelectron microscopic analyses (Stark etal., 2002; Valle et al., 2002, 2003a; Schuette etal., 2009; Villa etal., 2009), X-ray crystallographic analyses (Schmeing et al., 2009; Voorhees et al., 2010), and single-molecule observations (Blanchard et al., 2004; 
Lee etal., 2007; Geggier etal., 2010). These studies have identified a number of intermediate states of the sense codon decoding complex and have demonstrated that the selection of cognate aminoacyl-tRNA is achieved in two stages that are separated by irreversible GTP hydrolysis (Figure 4).

In this scheme, codon-anticodon interactions play a critical role in both the first and second stages of the sense codon decoding process. In the first stage, codon-anticodon interaction in the A site induces structural rearrangements of the $30 \mathrm{~S}$ ribosomal subunit; formation of the first and second base pairs expose the A1492 and A1493 residues from helix 44 of $16 \mathrm{~S}$ rRNA and the G530 residue of $16 \mathrm{~S}$ rRNA also undergoes a conformational change that enables it to interact with the second and third base pairs (Ogle et al., 2001). Moreover, conformation of the $30 \mathrm{~S}$ subunit is changed from an open to a closed form upon codon-anticodon binding (Ogle et al., 2002). These observations suggest that structural rearrangements at the decoding site are essential features of tRNA selection. It should be noted that similar structural rearrangements are also observed in the ribosome retaining near-cognate tRNA at the A site (Demeshkina et al., 2012). However, in this case, codon-anticodon interaction is destabilized and causes dissociation of tRNA from the ribosome. The stabilization of the codon-anticodon interaction in the ribosomal A site may promote

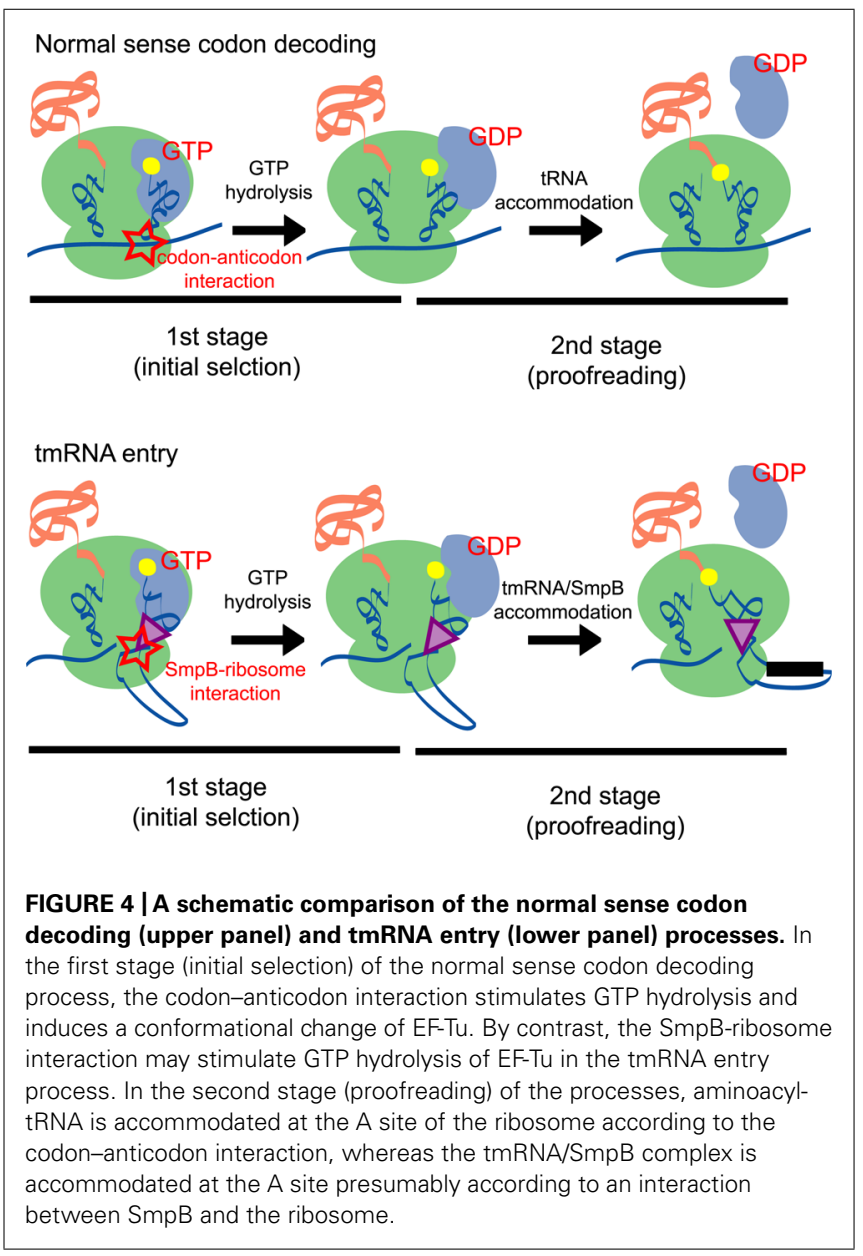

a kinked conformation of the tRNA structure and destabilize the interaction between the $3^{\prime}$ end of aminoacyl-tRNA and EF-Tu, thereby triggering GTP hydrolysis (Frank et al., 2005; Schmeing etal., 2009). In the second stage of the sense codon decoding process, codon-anticodon interactions support the so-called molecular spring movement of aminoacyl-tRNA that is released from its high-energy state (Frank et al., 2005; Sanbonmatsu et al., 2005; Whitford et al., 2010).

In contrast to the sense codon decoding process, codonanticodon interactions do not occur during tmRNA/SmpB entry because tmRNA does not possess an anticodon and the ribosome does not contain mRNA codon in its A site. Despite this situation, the tmRNA/SmpB complex binds to and facilitates the hydrolysis of EF-Tu (Barends et al., 2001; Valle et al., 2003b; Kaur et al., 2006) to enable its entry into the ribosomal A site (Shimizu and Ueda, 2006). Furthermore, the C-tail region of SmpB plays a crucial role in this process through an interaction with the decoding region of the $30 \mathrm{~S}$ ribosomal subunit, suggesting that the interaction between the ribosome and the $\mathrm{C}$-tail region of $\mathrm{SmpB}$ compensates for the absent codon-anticodon interaction.

The molecular mechanism of the compensation by SmpB has been revealed in part by the recently reported crystal structure of the complex composed of the T. thermophilus ribosome, a partial fragment of tmRNA, SmpB, and EF-Tu, which was trapped in the GDP form after GTP hydrolysis using the antibiotic kirromycin (Neubauer etal., 2012). In this structure, the C-tail region of SmpB forms an $\alpha$-helix as predicted (Miller et al., 2011); this region extends toward the mRNA path downstream of the A site to interact with the ribosomal protein S5. The structural rearrangements are similar with that of the ribosomal complex involved in canonical sense codon decoding, suggesting that binding of $\mathrm{SmpB}$ to the decoding region of the $30 \mathrm{~S}$ ribosomal subunit induces similar structural rearrangements in the ribosome, which may be necessary for the stimulation of GTP hydrolysis.

However, the exact mechanisms involved in the stimulation of GTP hydrolysis during tmRNA/SmpB entry may not be identical to the process that occurs during sense codon decoding. For example, mutations of the G530, A1492, and A1493 residues of 16S rRNA, which reduce the rates of peptidyl transfer and GTP hydrolysis in canonical decoding drastically, do not affect these reactions during the rescue of stalled ribosomes by tmRNA (Miller et al., 2011). In addition, the ribosomal protein S12, which has a stabilizing effect during canonical decoding, does not interact with SmpB (Neubauer et al., 2012). These findings may indicate that the interactions between the ribosome and the codonanticodon base pairs that occur during canonical decoding are not essential for structural rearrangements of the 30S ribosomal subunit and subsequent GTP hydrolysis. SmpB may use a different mechanism to compensate for the lack of codon-anticodon interaction in the A site of the ribosome to stimulate GTP hydrolysis.

Intriguingly, peptidyl-transfer rates are reduced markedly when the C-tail region of $\mathrm{SmpB}$ is mutated or deleted, although GTP hydrolysis rates are not affected (Miller et al., 2011). This finding suggests that the C-tail region may play a role after GTP hydrolysis, presumably in the step corresponding to the second proofreading 
stage in the canonical decoding process (Figure 4). Considering that key residues responsible for controlling the peptidyl-transfer rate are dispersed in the entire region of the C-tail (Sundermeier et al., 2005; Miller et al., 2011), interactions between these residues and several regions of the ribosome may play important roles in this process.

A number of questions related to the dynamics of accommodation of the tmRNA/SmpB complex into the ribosomal A site during the trans-translation process remain unanswered. For example, the mechanisms by which the ORF region of tmRNA is loaded into the A site and the resume codon in the ORF region is determined are still unknown. Although cryo-electron microscopic visualizations of the ribosome structure after translocation of the tmRNA to the P site (Fu et al., 2010; Weis etal., 2010; Ramrath et al., 2012) and mutational studies of both tmRNA and SmpB (Williams et al., 1999; Lee et al., 2001; Miller et al., 2008; Watts et al., 2009; Camenares et al., 2013) have added some insights, additional biochemical, structural, single-molecule imaging (Zhou et al., 2011), and simulative studies (Sanbonmatsu et al., 2005) are necessary to elucidate the precise dynamics of the trans-translation system on the ribosome.

\section{TWO ALTERNATIVE SYSTEMS FOR RIBOSOME RESCUE}

The trans-translation system has long been regarded as an exclusive method of ribosome rescue in bacteria. However, although it is essential for the viability of several phylogenetic groups (Huang et al., 2000; Glass et al., 2006; Ramadoss et al., 2013a), other groups, including E. coli, do not require the trans-translation system for survival (Komine et al., 1994; Karzai et al., 1999; Wiegert and Schumann, 2001), suggesting the existence of alternative pathways for stalled ribosome rescue. In fact, two alternative systems were recently identified: the ArfA/RF2 system and the ArfB system. The ribosome rescue mechanisms of these two pathways are described in the following sections.

\section{THE ArfA/RF2 SYSTEM}

ArfA (previously called YhdL) was originally identified as a protein factor essential for the viability of E. coli in the absence of the trans-translation system (Chadani et al., 2010). Using genetic screening, Chadani et al. (2010) determined that the arfA gene is required for suppression of the growth defect of cells lacking this system and showed that double depletion of ArfA and tmRNA from cells causes a loss of viability, indicating that ArfA plays a role complementary to that of the trans-translation system.

The E. coli arfA gene encodes a small protein of 72 amino acids. A conserved hairpin structure within the arfA mRNA (Schaub etal., 2012) is a target of RNase III and the ArfA protein is expressed from the truncated mRNA lacking an in-frame termination codon. Although the full-length ArfA polypeptide cannot be expressed in normal cells, the truncated form containing 53-55 of the N-terminal residues retains full functionality (Chadani et al., 2011a; Garza-Sánchez et al., 2011). When the trans-translation system is active in cells, the ribosome translating the arfA mRNA becomes a target of tmRNA/SmpB and the SsrA tag is attached to the synthesized ArfA peptide, leading to its subsequent degradation by SspB/ClpXP. Therefore, ArfA expression is regulated by the trans-translation system and may function as a backup system that substitutes for a deficient or suppressed trans-translation system.

Although ArfA can bind to the 50S subunit of the ribosome and peptidyl-tRNA in the ribosomal $\mathrm{P}$ site can be hydrolyzed in cell-free protein synthesis reaction mixtures containing S30 cell extract (Chadani et al., 2010), ArfA alone cannot hydrolyze peptidyl-tRNA on the purified ribosome complex, indicating the existence of factors that function cooperatively (Chadani et al., 2011b). Recent studies using a reconstituted cell-free protein synthesis system (Shimizu et al., 2001) showed that RF2, which catalyzes peptidyl-tRNA hydrolysis in the canonical termination step (Frolova et al., 1999; Song et al., 2000), also plays a catalytic role in peptidyl-tRNA hydrolysis during ArfA-dependent rescue of stalled ribosomes (Chadani et al., 2012; Shimizu, 2012). Mutation of the GGQ amino acid motif in RF2 disrupts this process (Chadani et al., 2012). RF2 can bind to stalled ribosomes in the presence of ArfA, whereas RF1 is unable to bind to ribosomes in the presence or absence of ArfA (Shimizu, 2012), indicating that ArfA recruits RF2 but not RF1 into the A site of the stalled ribosome, where it promotes peptidyl-tRNA hydrolysis (Figure 5).

The mechanism by which ArfA recruits RF2 into the stalled ribosome is still unknown. A18T-mutated ArfA, which suppresses ArfA-dependent ribosome rescue (Chadani et al., 2010), is unable to support peptidyl-tRNA hydrolysis in vitro. However, this mutation does not inhibit binding of RF2 to the ribosome (Shimizu, 2012), suggesting that simply recruiting RF2 into the stalled ribosome is not sufficient for the ArfAdependent ribosome rescue system. The mechanism by which

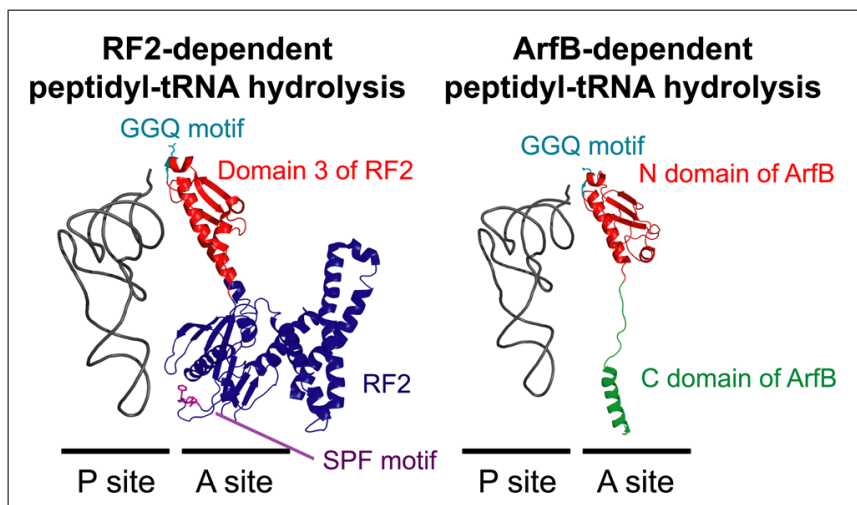

FIGURE 5 | A structural comparison of RF2-dependent (left panel) and ArfB-dependent (right panel) peptidyl-tRNA hydrolysis on the ribosome. In the normal translation process, RF2 binds to the ribosomal A site according to the interaction between the termination codon and the SPF motif (purple) in RF2. In the ArfA/RF2 ribosome rescue process, RF2 may bind to the A site similarly, albeit in a codon-independent and ArfA-dependent manner. ArfB binds to the A site according to the interaction between the $\mathrm{C}$ domain of $\mathrm{ArfB}$ (green) and the ribosome in a codon-independent manner. In both reactions, the hydrolysis of peptidyl-tRNA in the $P$ site is catalyzed by a specific GGQ amino acid motif (light blue) in the conserved domain of RF2 (domain 3; red) or ArfB (N domain; red). The structural coordinates of the RF2-dependent and ArfB-dependent hydrolysis complexes were taken from PDB entries 3F1E (Korostelev et al., 2008) and 4DH9 (Gagnon et al., 2012), respectively. 
ArfA selectively recruits RF2 but not RF1 is also intriguing because the primary sequences and structures of RF1 and RF2 on the ribosome are very similar (Laurberg et al., 2008; Korostelev et al., 2008; Korostelev, 2011). Elucidating these mechanisms will contribute to understanding the protein synthesis system and the basis for bacterial evolution, and may also be useful for antibiotic development (Ramadoss et al., 2013b) because neither the trans-translation system nor the ArfA systems are found in animals.

\section{THE ArfB SYSTEM}

Evidence that ArfB (previously called YaeJ) plays a role in stalled ribosome rescue was reported independently by two research groups (Chadani et al., 2011b; Handa et al., 2011). Handa et al. (2011) demonstrated that ArfB hydrolyzes peptidyl-tRNA on the ribosome in vitro and Chadani et al. (2011b) genetically identified $\mathrm{ArfB}$ as a multicopy suppressor of the growth defect of ssrA/arfA double mutants and then showed similar results to those reported by Handa et al. (2011). Notably, immature colon carcinoma transcript 1 (ICT1), an ArfB homolog present in the human mitochondrial ribosome complex, also displays ribosomedependent peptidyl-tRNA hydrolysis activity (Richter et al., 2010), suggesting that ICT1 plays a role in stalled ribosome rescue in human mitochondria.

Compared with the trans-translation and ArfA/RF2 systems, the molecular mechanism of ArfB-dependent ribosome rescue is relatively simple. ArfB consists of a C-terminal unstructured region and a globular $\mathrm{N}$-terminal domain containing a GGQ amino acid motif that resembles domain 3 of class-1 RFs. Analysis of the crystal structure of $E$. coli ArfB bound to the $T$. thermophilus ribosome with the initiator tRNA ${ }^{\mathrm{fMet}}$ (Gagnon et al., 2012) showed that the N-terminal domain is located in the A site of the 50S ribosomal subunit and the GGQ motif is positioned in the peptidyl-transferase center adjacent to the CCA end of the initiator tRNA ${ }^{\text {fMet }}$ in the ribosomal $\mathrm{P}$ site. Together with the finding that mutation of the GGQ motif in ArfB inhibits its peptidyl-tRNA hydrolysis activity (Chadani et al., 2011b; Handa et al., 2011), the structure indicates that this motif is responsible for peptidyl-tRNA hydrolysis to rescue stalled ribosomes.

In addition to mutation of the $\mathrm{N}$-terminal domain, truncation of the C-terminal unstructured region of $\mathrm{ArfB}$ also suppresses peptidyl-tRNA hydrolysis on the ribosome (Chadani et al., 2011b; Handa et al., 2011). Analysis of the crystal structure showed that, similar to the C-tail region of SmpB in the tmRNA entry process of the trans-translation system (Neubauer et al., 2012), the C-terminal unstructured region of ArfB forms an $\alpha$-helix and is accommodated inside the mRNA path downstream of the A site (Gagnon et al., 2012). Several point mutations of basic residues in the $\mathrm{C}$-terminal region suppress the activity of $\mathrm{ArfB}$ in vitro, indicating that the interaction between the mRNA path and these residues plays an important role in ArfB-mediated ribosome rescue (Kogure et al., 2014). In summary, ArfB plays an independent role in stalled ribosome rescue by interacting with the A site of the ribosome and catalyzing the hydrolysis of peptidyl-tRNA in the $\mathrm{P}$ site in a codon-independent manner (Figure 5).

\section{ESSENTIAL FEATURES OF THE RIBOSOMAL SITUATION REQUIRED FOR PROPER FUNCTIONING OF THE THREE RESCUE SYSTEMS}

A common feature of the three ribosome rescue pathways described here is that they may be structurally designed to selectively target stalled ribosomes lacking a codon in the A site. The $\mathrm{C}$-tail regions of SmpB and $\mathrm{ArfB}$ are both accommodated in the mRNA path downstream of the ribosomal A site (Gagnon et al., 2012; Neubauer et al., 2012), suggesting that binding of these proteins is inhibited if an mRNA is located downstream of the A site, which is a common feature of translating ribosomes. The structure of the ribosome complex containing ArfA and RF2 has not yet been clarified; however, since the ArfA/RF2 system uses RF2 for peptidyl-tRNA hydrolysis, binding of RF2 to the A site may be limited to ribosomes in which the A site is either unoccupied or occupied by a UAA or UGA codon, which matches the peptide anticodon region (SPF motif) of RF2 (Ito et al., 2000).

Accordingly, in vitro studies showed that the presence of an mRNA downstream of the A site inhibits the three ribosome rescue pathways. An in vitro study of the trans-translation system showed that the rate of alanyl-transfer to the nascent polypeptide is inversely proportional to the length of the mRNA downstream of the A site (Ivanova et al., 2004). Furthermore, ribosome rescue by the ArfA/RF2 system is reduced significantly if the stalled ribosome contains additional mRNA sequences downstream of the A site (Shimizu, 2012). The ribosome rescue activity of the ArfB system is also inversely proportional to the length of mRNA downstream of the A site, although it is maintained when a sufficient length of mRNA, which fully covers the mRNA path downstream of the A site on the ribosome, remains on the ribosome (Shimizu, 2012). The fact that ArfB is able to promote ribosome rescue in the presence of a sufficient length of mRNA is in agreement with the finding that ArfB can hydrolyze peptidyl-tRNA on ribosomes stalled by a rare codon cluster in vitro (Handa et al., 2011). These studies may suggest that the flexibility of mRNA allows the C-terminal region of ArfB to bind to the ribosome by avoiding a steric clash (Gagnon et al., 2012).

The in vitro studies described above suggest that the ArfB system may interfere with the normal translation system because it can cover a wider range of ribosomal situations than the transtranslation or ArfA/RF2 systems. There is currently no explanation for the broader specificity of the ArfB system. Notably, endogenous ArfB cannot restore the viability of $E$. coli cells depleted of both ArfA and tmRNA, whereas overexpression of exogenous ArfB does restore this defect (Chadani etal., 2011b). This lack of compensation may be due to the lower expression level of ArfB compared to SmpB in E. coli (about 0.5 and 15 protein molecules/cell, respectively; Taniguchi et al., 2010), suggesting that ArfB may not play a crucial role in ribosome rescue in E. coli cells. However, the situation may be different in other bacterial cells because only a subset of $\beta$ - and $\gamma$-proteobacteria contains ArfA homologs (Schaub et al., 2012). The tight regulation of ArfA expression by the trans-translation system suggests that these two systems have complementary roles in ribosome rescue (Chadani et al., 2011a; Garza-Sánchez et al., 2011), perhaps indicating that 
they play major roles in the ribosome rescue process in E. coli cells at least. Therefore, it is reasonable to speculate that the transtranslation and ArfA/RF2 systems are structurally designed to target stalled ribosomes more precisely.

In vivo studies showed that the trans-translation system targets ribosomes that are stalled at certain regions of mRNA due to amino acid starvation (Garza-Sánchez et al., 2008; Li et al., 2008) or the presence of a rare codon cluster (Roche and Sauer, 1999) or specific sequence that causes elongation arrest (Sunohara etal., 2004), in which a sufficient length of mRNA may remain on the ribosome downstream of the A site. These observations may not contradict the in vitro studies because such pausing reportedly induces cleavage of the A site mRNA codon (Hayes and Sauer, 2003; Yamamoto et al., 2003; Sunohara et al., 2004; Garza-Sánchez et al., 2008). The mechanisms involved in this cleavage process are largely unknown, although RNase II exonuclease activity has been implicated (Garza-Sánchez et al., 2009). Additional studies of the ways in which cells handle the mRNA portion of stalled ribosomes by controlling RNase activity are necessary to understand the essential features of the ribosomal situation required for proper functioning of the rescue systems.

\section{ACKNOWLEDGMENT}

This work was supported by the Quantitative Biology Center RIKEN.

\section{REFERENCES}

Abo, T., Inada, T., Ogawa, K., and Aiba, H. (2000). SsrA-mediated tagging and proteolysis of LacI and its role in the regulation of lac operon. EMBO J. 19, 3762-3729. doi: 10.1093/emboj/19.14.3762

Barends, S., Karzai, A. W., Sauer, R. T., Wower, J., and Kraal, B. (2001). Simultaneous and functional binding of SmpB and EF-Tu-TP to the alanyl acceptor arm of tmRNA. J. Mol. Biol. 314, 9-21. doi: 10.1006/jmbi.2001.5114

Bessho, Y., Shibata, R., Sekine, S., Murayama, K., Higashijima, K., Hori-Takemoto, C., et al. (2007). Structural basis for functional mimicry of long-variable-arm tRNA by transfer-messenger RNA. Proc. Natl. Acad. Sci. U.S.A. 104, 8293-8298. doi: 10.1073/pnas.0700402104

Blanchard, S. C., Gonzalez, R. L., Kim, H. D., Chu, S., and Puglisi, J. D. (2004). tRNA selection and kinetic proofreading in translation. Nat. Struct. Mol. Biol. 11, 1008-1014. doi: 10.1038/nsmb831

Camenares, D., Dulebohn, D. P., Svetlanov, A., and Karzai, A. W. (2013). Active and accurate trans-translation requires distinct determinants in the $\mathrm{C}$-terminal tail of SmpB protein and the mRNA-like domain of transfer messenger RNA (tmRNA). J. Biol. Chem. 288, 30527-30542. doi: 10.1074/jbc.M1 13.503896

Chadani, Y., Ito, K., Kutsukake, K., and Abo, T. (2012). ArfA recruits release factor 2 to rescue stalled ribosomes by peptidyl-tRNA hydrolysis in Escherichia coli. Mol. Microbiol. 86, 37-50. doi: 10.1111/j.1365-2958.2012.08190.x

Chadani, Y., Ono, K., Ozawa, S., Takahashi, Y., Takai, K., Nanamiya, H., et al. (2010). Ribosome rescue by Escherichia coli ArfA (YhdL) in the absence of trans-translation system. Mol. Microbiol. 78, 796-808. doi: 10.1111/j.13652958.2010.07375.x

Chadani, Y., Matsumoto, E., Aso, H., Wada, T., Kutsukake, K., Sutou, S., et al. (2011a). trans-translation-mediated tight regulation of the expression of the alternative ribosome-rescue factor ArfA in Escherichia coli. Genes Genet. Syst. 86, 151-163. doi: 10.1266/ggs.86.151

Chadani, Y., Ono, K., Kutsukake, K., and Abo, T. (2011b). Escherichia coli YaeJ protein mediates a novel ribosome-rescue pathway distinct from SsrA- and ArfA-mediated pathways. Mol. Microbiol. 80, 772-785. doi: 10.1111/j.13652958.2011.07607.x

Daviter, T., Gromadski, K. B., and Rodnina, M. V. (2006). The ribosome's response to codon-anticodon mismatches. Biochimie 88, 1001-1011. doi: 10.1016/j.biochi.2006.04.013
Demeshkina, N., Jenner, L., Westhof, E., Yusupov, M., and Yusupova, G. (2012). A new understanding of the decoding principle on the ribosome. Nature 484, 256-259. doi: 10.1038/nature10913

Dong, G., Nowakowski, J., and Hoffman, D. W. (2002). Structure of small protein B: the protein component of the tmRNA-SmpB system for ribosome rescue. EMBO J. 21, 1845-1854. doi: 10.1093/emboj/21.7.1845

Draper, D. E., and Reynaldo, L. P. (1999). RNA binding strategies of ribosomal proteins. Nucleic Acids Res. 27, 381-388. doi: 10.1093/nar/27.2.381

Felden, B., Hanawa, K., Atkins, J. F., Himeno, H., Muto, A., Gesteland, R. F., et al. (1998). Presence and location of modified nucleotides in Escherichia coli tmRNA: structural mimicry with tRNA acceptor branches. EMBO J. 17, 3188-3196. doi: 10.1093/emboj/17.11.3188

Francklyn, C., and Schimmel, P. (1989). Aminoacylation of RNA minihelices with alanine. Nature 337, 478-481. doi: 10.1038/337478a0

Frank, J., Sengupta, J., Gao, H., Li, W., Valle, M., Zavialov, A., et al. (2005). The role of tRNA as a molecular spring in decoding, accommodation, and peptidyl transfer. FEBS Lett. 579, 959-962. doi: 10.1016/j.febslet.2004.10.105

Frolova, L. Y., Tsivkovskii, R. Y., Sivolobova, G. F., Oparina, N. Y., Serpinsky, O. I., Blinov, V. M., et al. (1999). Mutations in the highly conserved GGQ motif of class 1 polypeptide release factors abolish ability of human eRF1 to trigger peptidyl-tRNA hydrolysis. RNA 5, 1014-1020. doi: 10.1017/S135583829999043X

Fu, J., Hashem, Y., Wower, I., Lei, J., Liao, H. Y., Zwieb, C., et al. (2010). Visualizing the transfer-messenger RNA as the ribosome resumes translation. EMBO J. 29, 3819-3825. doi: 10.1038/emboj.2010.255

Gagnon, M. G., Seetharaman, S. V., Bulkley, D., and Steitz, T. A. (2012). Structural basis for the rescue of stalled ribosomes: structure of Yaej bound to the ribosome. Science 335, 1370-1372. doi: 10.1126/science. 1217443

Garza-Sánchez, F., Gin, J. G., and Hayes, C. S. (2008). Amino acid starvation and colicin D treatment induce A-site mRNA cleavage in Escherichia coli. J. Mol. Biol. 378, 505-519. doi: 10.1016/j.jmb.2008.02.065

Garza-Sánchez, F., Schaub, R. E., Janssen, B. D., and Hayes, C. S. (2011). tmRNA regulates synthesis of the ArfA ribosome rescue factor. Mol. Microbiol. 80, 12041219. doi: $10.1111 /$ j.1365-2958.2011.07638.x

Garza-Sánchez, F., Shoji, S., Fredrick, K., and Hayes, C. S. (2009). RNase II is important for A-site mRNA cleavage during ribosome pausing. Mol. Microbiol. 73, 882-897. doi: 10.1111/j.1365-2958.2009.06813.x

Geggier, P., Dave, R., Feldman, M. B., Terry, D. S., Altman, R. B., Munro, J. B., et al. (2010). Conformational sampling of aminoacyl-tRNA during selection on the bacterial ribosome. J. Mol. Biol. 399, 576-595. doi: 10.1016/j.jmb.2010.04.038

Glass, J. I., Assad-Garcia, N., Alperovich, N., Yooseph, S., Lewis, M. R., Maruf, M., et al. (2006). Essential genes of a minimal bacterium. Proc. Natl. Acad. Sci. U.S.A. 103, 425-430. doi: 10.1073/pnas.0510013103

Gottesman, S., Roche, E., Zhou, Y., and Sauer, R. T. (1998). The ClpXP and ClpAP proteases degrade proteins with carboxy-terminal peptide tails added by the SsrAtagging system. Genes Dev. 12, 1338-1347. doi: 10.1101/gad.12.9.1338

Hanawa-Suetsugu, K., Takagi, M., Inokuchi, H., Himeno, H., and Muto, A. (2002). SmpB functions in various steps of trans-translation. Nucleic Acids Res. 30, 16201629. doi: $10.1093 / \mathrm{nar} / 30.7 .1620$

Handa, Y., Inaho, N., and Nameki, N. (2011). YaeJ is a novel ribosome-associated protein in Escherichia coli that can hydrolyze peptidyl-tRNA on stalled ribosomes. Nucleic Acids Res. 39, 1739-1748. doi: 10.1093/nar/gkq1097

Hayes, C. S., and Sauer, R. T. (2003). Cleavage of the A site mRNA codon during ribosome pausing provides a mechanism for translational quality control. Mol. Cell 12, 903-911. doi: 10.1016/S1097-2765(03)00385-X

Hou, Y. M., and Schimmel, P. (1988). A simple structural feature is a major determinant of the identity of a transfer RNA. Nature 333, 140-145. doi: $10.1038 / 333140 \mathrm{a} 0$

Huang, C., Wolfgang, M. C., Withey, J., Koomey, M., and Friedman, D. I. (2000). Charged tmRNA but not tmRNA-mediated proteolysis is essential for Neisseria gonorrhoeae viability. EMBO J. 19, 1098-1107. doi: 10.1093/emboj/19.5.1098

Ito, K., Uno, M., and Nakamura, Y. (2000). A tripeptide 'anticodon' deciphers stop codons in messenger RNA. Nature 403, 680-684. doi: 10.1038/35001115

Ivanova, N., Pavlov, M. Y., Felden, B., and Ehrenberg, M. (2004). Ribosome rescue by tmRNA requires truncated mRNAs. J. Mol. Biol. 338, 33-41. doi: 10.1016/j.jmb.2004.02.043

Jacob, Y., Sharkady, S. M., Bhardwaj, K., Sanda, A., and Williams, K. P. (2005). Function of the SmpB tail in transfer-messenger RNA translation revealed by a nucleus-encoded form. J. Biol. Chem. 280, 5503-5509. doi: 10.1074/jbc.M409277200 
Karzai, A. W., Susskind, M. M., and Sauer, R. T. (1999). SmpB, a unique RNA-binding protein essential for the peptide-tagging activity of SsrA (tmRNA). EMBO J. 18, 3793-3799. doi: 10.1093/emboj/18.13.3793

Kaur, S., Gillet, R., Li, W., Gursky, R., and Frank, J. (2006). Cryo-EM visualization of transfer messenger RNA with two SmpBs in a stalled ribosome. Proc. Natl. Acad. Sci. U.S.A. 103, 16484-16489. doi: 10.1073/pnas.0607438103

Kim, Y. I., Burton, R. E., Burton, B. M., Sauer, R. T., and Baker, T. A. (2000). Dynamics of substrate denaturation and translocation by the ClpXP degradation machine. Mol. Cell 5, 639-648. doi: 10.1016/S1097-2765(00) 80243-9

Kobayashi, K., Kuwana, R., and Takamatsu, H. (2008). kinA mRNA is missing a stop codon in the undomesticated Bacillus subtilis strain ATCC 6051. Microbiology 154, 54-63. doi: 10.1099/mic.0.2007/011783-0

Kogure, H., Handa, Y., Nagata, M., Kanai, N., Güntert, P., Kubota, K., et al. (2014). Identification of residues required for stalled-ribosome rescue in the codon-independent release factor YaeJ. Nucleic Acids Res. 42, 3152-3163. doi: $10.1093 /$ nar/gkt1280

Komine, Y., Kitabatake, M., Yokogawa, T., Nishikawa, K., and Inokuchi, H. (1994). A tRNA-like structure is present in 10Sa RNA, a small stable RNA from Escherichia coli. Proc. Natl. Acad. Sci. U.S.A. 91, 9223-9227. doi: 10.1073/pnas.91.20.9223

Korostelev, A. A. (2011). Structural aspects of translation termination on the ribosome. RNA 17, 1409-1421. doi: 10.1261/rna.2733411

Korostelev, A., Asahara, H., Lancaster, L., Laurberg, M., Hirschi, A., Zhu, J., et al. (2008). Crystal structure of a translation termination complex formed with release factor RF2. Proc. Natl. Acad. Sci. U.S.A. 105, 19684-19689. doi: 10.1073/pnas.0810953105

Laurberg, M., Asahara, H., Korostelev, A., Zhu, J., Trakhanov, S., and Noller, H. F. (2008). Structural basis for translation termination on the 70 S ribosome. Nature 454, 852-857. doi: 10.1038/nature07115

Lee, S., Ishii, M., Tadaki, T., Muto, A., and Himeno, H. (2001). Determinants on tmRNA for initiating efficient and precise trans-translation: some mutations upstream of the tag-encoding sequence of Escherichia coli tmRNA shift the initiation point of trans-translation in vitro. RNA 7, 999-1012.

Lee, T. H., Blanchard, S. C., Kim, H. D., Puglisi, J. D., and Chu, S. (2007). The role of fluctuations in tRNA selection by the ribosome. Proc. Natl. Acad. Sci. U.S.A. 104, 13661-13665. doi: 10.1073/pnas.0705988104

Levchenko, I., Seidel, M., Sauer, R. T., and Baker, T. A. (2000). A specificityenhancing factor for the ClpXP degradation machine. Science 289, 2354-2356. doi: $10.1126 /$ science.289.5488.2354

Lies, M., and Maurizi, M. R. (2008). Turnover of endogenous SsrA-tagged proteins mediated by ATP-dependent proteases in Escherichia coli. J. Biol. Chem. 283, 22918-22929. doi: 10.1074/jbc.M801692200

Li, X., Yagi, M., Morita, T., and Aiba, H. (2008). Cleavage of mRNAs and role of tmRNA system under amino acid starvation in Escherichia coli. Mol. Microbiol. 68, 462-473. doi: 10.1111/j.1365-2958.2008.06167.x

Miller, M. R., Healey, D. W., Robison, S. G., Dewey, J. D., and Buskirk, A. R. (2008). The role of upstream sequences in selecting the reading frame on tmRNA. BMC Biol. 6:29. doi: 10.1186/1741-7007-6-29

Miller, M. R., Liu, Z., Cazier, D. J., Gebhard, G. M., Herron, S. R., Zaher, H. S., et al. (2011). The role of SmpB and the ribosomal decoding center in licensing tmRNA entry into stalled ribosomes. RNA 17, 1727-1736. doi: 10.1261/rna.2821711

Murzin, A. G. (1993). OB(oligonucleotide/oligosaccharide binding)-fold: common structural and functional solution for non-homologous sequences. EMBO J. 12 861-867.

Nameki, N., Felden, B., Atkins, J. F., Gesteland, R. F., Himeno, H., and Muto, A (1999). Functional and structural analysis of a pseudoknot upstream of the tag-encoded sequence in E. coli tmRNA. J. Mol. Biol. 286, 733-744. doi: 10.1006/jmbi.1998.2487

Nameki, N., Tadaki, T., Himeno, H., and Muto, A. (2000). Three of four pseudoknots in tmRNA are interchangeable and are substitutable with single-stranded RNAs. FEBS Lett. 470, 345-349. doi: 10.1016/S0014-5793(00)01349-1

Neubauer, C., Gillet, R., Kelley, A. C., and Ramakrishnan, V. (2012). Decoding in the absence of a codon by tmRNA and SmpB in the ribosome. Science 335, 1366-1369. doi: 10.1126/science.1217039

Nissen, P., Kjeldgaard, M., Thirup, S., Polekhina, G., Reshetnikova, L., Clark, B. F., et al. (1995). Crystal structure of the ternary complex of Phe-tRNAPhe, EF-Tu, and a GTP analog. Science 270, 1464-1472. doi: 10.1126/science.270. 5241.1464
Nissen, P., Thirup, S., Kjeldgaard, M., and Nyborg, J. (1999). The crystal structure of Cys-tRNACys-EF-Tu-GDPNP reveals general and specific features in the ternary complex and in tRNA. Structure 7, 143-156. doi: 10.1016/S0969-2126(99)80021-5

Ogle, J. M., Brodersen, D. E., Clemons, W. M. Jr., Tarry, M. J., Carter, A. P., and Ramakrishnan, V. (2001). Recognition of cognate transfer RNA by the 30 S ribosomal subunit. Science 292, 897-902. doi: 10.1126/science. 1060612

Ogle, J. M., Murphy, F. V., Tarry, M. J., and Ramakrishnan, V. (2002). Selection of tRNA by the ribosome requires a transition from an open to a closed form. Cell 111, 721-732. doi: 10.1016/S0092-8674(02)01086-3

Ramadoss, N. S., Zhou, X., and Keiler, K. C. (2013a). tmRNA is essential in Shigella flexneri. PLoS ONE 8:e57537. doi: 10.1371/journal.pone.0057537

Ramadoss, N. S., Alumasa, J. N., Cheng, L., Wang, Y., Li, S., Chambers, B. S., et al. (2013b). Small molecule inhibitors of trans-translation have broadspectrum antibiotic activity. Proc. Natl. Acad. Sci. U.S.A. 110, 10282-10287. doi: $10.1073 /$ pnas. 1302816110

Ramrath, D. J., Yamamoto, H., Rother, K., Wittek, D., Pech, M., Mielke, T., et al. (2012). The complex of tmRNA-SmpB and EF-G on translocating ribosomes. Nature 485, 526-529. doi: 10.1038/nature11006

Richter, R., Rorbach, J., Pajak, A., Smith, P. M., Wessels, H. J., Huynen, M. A., et al. (2010). A functional peptidyl-tRNA hydrolase, ICT1, has been recruited into the human mitochondrial ribosome. EMBO J. 29, 1116-1125. doi: 10.1038/emboj.2010.14

Roche, E. D., and Sauer, R. T. (1999). SsrA-mediated peptide tagging caused by rare codons and tRNA scarcity. EMBO J. 18, 4579-4589. doi: 10.1093/emboj/18.16.4579

Rodnina, M. V., and Wintermeyer, W. (2001). Fidelity of aminoacyl-tRNA selection on the ribosome: kinetic and structural mechanisms. Annu. Rev. Biochem. 70, 415-435. doi: 10.1146/annurev.biochem.70.1.415

Sanbonmatsu, K. Y., Joseph, S., and Tung, C. S. (2005). Simulating movement of tRNA into the ribosome during decoding. Proc. Natl. Acad. Sci. U.S.A. 102, 15854-15859. doi: 10.1073/pnas.0503456102

Schaub, R. E., Poole, S. J., Garza-Sánchez, F., Benbow, S., and Hayes, C. S. (2012). Proteobacterial ArfA peptides are synthesized from non-stop messenger RNAs. J. Biol. Chem. 287, 29765-29775. doi: 10.1074/jbc.M112.374074

Schmeing, T. M., Voorhees, R. M., Kelley, A. C., Gao, Y. G., Murphy, F. V. IV, Weir, J. R., etal. (2009). The crystal structure of the ribosome bound to EF-Tu and aminoacyl-tRNA. Science 326, 688-694. doi: 10.1126/science. 1179700

Schuette, J. C., Murphy, F. V. IV, Kelley, A. C., Weir, J. R., Giesebrecht, J., Connell, S. R., et al. (2009). GTPase activation of elongation factor EF-Tu by the ribosome during decoding. EMBO J. 28, 755-765. doi: 10.1038/emboj.2009.26

Shimizu, Y. (2012). ArfA recruits RF2 into stalled ribosomes. J. Mol. Biol. 423, 624-631. doi: 10.1016/j.jmb.2012.08.007

Shimizu, Y., Inoue, A., Tomari, Y., Suzuki, T., Yokogawa, T., Nishikawa, K., et al. (2001). Cell-free translation reconstituted with purified components. Nat. Biotechnol. 19, 751-755. doi: 10.1038/90802

Shimizu, Y., and Ueda, T. (2002). The role of SmpB protein in trans-translation. FEBS Lett. 514, 74-77. doi: 10.1016/S0014-5793(02)02333-5

Shimizu, Y., and Ueda, T. (2006). SmpB triggers GTP hydrolysis of elongation factor Tu on ribosomes by compensating for the lack of codon-anticodon interaction during trans-translation initiation. J. Biol. Chem. 281, 15987-15996. doi: 10.1074/jbc.M512165200

Someya, T., Nameki, N., Hosoi, H., Suzuki, S., Hatanaka, H., Fujii, M., et al. (2003). Solution structure of a tmRNA-binding protein, $\mathrm{SmpB}$, from Thermus thermophilus. FEBS Lett. 535, 94-100. doi: 10.1016/S0014-5793(02)03880-2

Song, H., Mugnier, P., Das, A. K., Webb, H. M., Evans, D. R., Tuite, M. F., et al. (2000). The crystal structure of human eukaryotic release factor eRF1-mechanism of stop codon recognition and peptidyl-tRNA hydrolysis. Cell 100, 311-321. doi: 10.1016/S0092-8674(00)80667-4

Stark, H., Rodnina, M. V., Wieden, H. J., Zemlin, F., Wintermeyer, W., and van Heel, M. (2002). Ribosome interactions of aminoacyl-tRNA and elongation factor $\mathrm{Tu}$ in the codon-recognition complex. Nat. Struct. Biol. 9, 849-854. doi: $10.1038 /$ nsb859

Sundermeier, T. R., Dulebohn, D. P., Cho, H. J., and Karzai, A. W. (2005). A previously uncharacterized role for small protein $\mathrm{B}(\mathrm{SmpB})$ in transfer messenger RNA-mediated trans-translation. Proc. Natl. Acad. Sci. U.S.A. 102, 2316-23121. doi: 10.1073/pnas.0409694102 
Sunohara, T., Jojima, K., Tagami, H., Inada, T., and Aiba, H. (2004). Ribosome stalling during translation elongation induces cleavage of mRNA being translated in Escherichia coli. J. Biol. Chem. 279, 15368-15375. doi: 10.1074/jbc.M312 805200

Swairjo, M. A., Otero, F. J., Yang, X. L., Lovato, M. A., Skene, R. J., McRee, D. E., et al. (2004). Alanyl-tRNA synthetase crystal structure and design for acceptor-stem recognition. Mol. Cell 13, 829-841. doi: 10.1016/S1097-2765(04)00126-1

Taniguchi, Y., Choi, P. J., Li, G. W., Chen, H., Babu, M., Hearn, J., et al. (2010). Quantifying E. coli proteome and transcriptome with single-molecule sensitivity in single cells. Science 329, 533-538. doi: 10.1126/science.1188308

Tanner, D. R., Dewey, J. D., Miller, M. R., and Buskirk, A. R. (2006). Genetic analysis of the structure and function of transfer messenger RNA pseudoknot 1. J. Biol. Chem. 281, 10561-10566. doi: 10.1074/jbc.M600167200

Williams, K. P., Martindale, K. A., and Bartel, D. P. (1999). Resuming translation on tmRNA: a unique mode of determining a reading frame. EMBO J. 18, 5423-5433. doi: 10.1093/emboj/18.19.5423

Wower, I. K., Zwieb, C., and Wower, J. (2004). Contributions of pseudoknots and protein $\mathrm{SmpB}$ to the structure and function of tmRNA in trans-translation. $J$. Biol. Chem. 279, 54202-54209. doi: 10.1074/jbc.M410488200

Wower, I. K., Zwieb, C., and Wower, J. (2009). Escherichia coli tmRNA lacking pseudoknot 1 tags truncated proteins in vivo and in vitro. RNA 15, 128-137. doi: 10.1261/rna.1192409

Ueda, K., Yamamoto, Y., Ogawa, K., Abo, T., Inokuchi, H., and Aiba, H. (2002). Bacterial SsrA system plays a role in coping with unwanted translational readthrough caused by suppressor tRNAs. Genes Cells 7, 509-519. doi: 10.1046/j.1365-2443.2002.00537.x

Valle, M., Sengupta, J., Swami, N. K., Grassucci, R. A., Burkhardt, N., Nierhaus, K. H., et al. (2002). Cryo-EM reveals an active role for aminoacyl-tRNA in the accommodation process. EMBO J. 21, 3557-3567. doi: 10.1093/emboj/cdf326

Valle, M., Zavialov, A., Li, W., Stagg, S. M., Sengupta, J., Nielsen, R. C., et al. (2003a). Incorporation of aminoacyl-tRNA into the ribosome as seen by cryo-electron microscopy. Nat. Struct. Biol. 10, 899-906. doi: 10.1038/nsb1003

Valle, M., Gillet, R., Kaur, S., Henne, A., Ramakrishnan, V., and Frank, J. (2003b). Visualizing tmRNA entry into a stalled ribosome. Science 300, 127-130. doi: 10.1126/science. 1081798

Villa, E., Sengupta, J., Trabuco, L. G., LeBarron, J., Baxter, W. T., Shaikh, T. R., et al. (2009). Ribosome-induced changes in elongation factor Tu conformation control GTP hydrolysis. Proc. Natl. Acad. Sci. U.S.A. 106, 1063-1068. doi: 10.1073/pnas.0811370106
Voorhees, R. M., Schmeing, T. M., Kelley, A. C., and Ramakrishnan, V. (2010). The mechanism for activation of GTP hydrolysis on the ribosome. Science 330, 835-838. doi: 10.1126/science.1194460

Watts, T., Cazier, D., Healey, D., and Buskirk, A. (2009). SmpB contributes to reading frame selection in the translation of transfer-messenger RNA. J. Mol. Biol. 391, 275-281. doi: 10.1016/j.jmb.2009.06.037

Weis, F., Bron, P., Giudice, E., Rolland, J. P., Thomas, D., Felden, B., et al. (2010). tmRNA-SmpB: a journey to the centre of the bacterial ribosome. EMBO J. 29, 3810-3818. doi: 10.1038/emboj.2010.252

Wiegert, T., and Schumann, W. (2001). SsrA-mediated tagging in Bacillus subtilis. J. Bacteriol. 183, 3885-3889. doi: 10.1128/JB.183.13.3885-3889.2001

Whitford, P. C., Onuchic, J. N., and Sanbonmatsu, K. Y. (2010). Connecting energy landscapes with experimental rates for aminoacyl-tRNA accommodation in the ribosome. J. Am. Chem. Soc. 132, 13170-13171. doi: 10.1021/ja1061399

Yamamoto, Y., Sunohara, T., Jojima, K., Inada, T., and Aiba, H. (2003). SsrA-mediated trans-translation plays a role in mRNA quality control by facilitating degradation of truncated mRNAs. RNA 9, 408-418. doi: 10.1261/rna. 2174803

Zhou, Z. P., Shimizu, Y., Tadakuma, H., Taguchi, H., Ito, K., and Ueda, T. (2011). Single molecule imaging of the trans-translation entry process via anchoring of the tagged ribosome. J. Biochem. 149, 609-618. doi: 10.1093/jb/mvr010

Conflict of Interest Statement: The author declares that the research was conducted in the absence of any commercial or financial relationships that could be construed as a potential conflict of interest.

Received: 18 December 2013; accepted: 28 March 2014; published online: 10 April 2014.

Citation: Shimizu Y (2014) Biochemical aspects of bacterial strategies for handling the incomplete translation processes. Front. Microbiol. 5:170. doi: 10.3389/fmicb.2014. 00170

This article was submitted to Microbial Physiology and Metabolism, a section of the journal Frontiers in Microbiology.

Copyright (c) 2014 Shimizu. This is an open-access article distributed under the terms of the Creative Commons Attribution License (CC BY). The use, distribution or reproduction in other forums is permitted, provided the original author(s) or licensor are credited and that the original publication in this journal is cited, in accordance with accepted academic practice. No use, distribution or reproduction is permitted which does not comply with these terms. 